\title{
Nonexposure endoscopic full-thickness resection with two flexible endoscopes equipped with a suturing device: ex vivo study
}

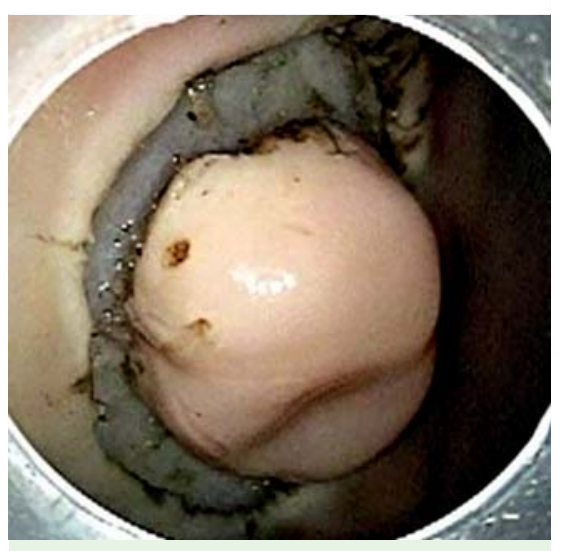

Fig. 1 After a pseudolesion with a 40-mm diameter has been marked, a circular ring with a width of $8 \mathrm{~mm}$ reaching the muscularis propria is made by using the endoscopic submucosal dissection technique.

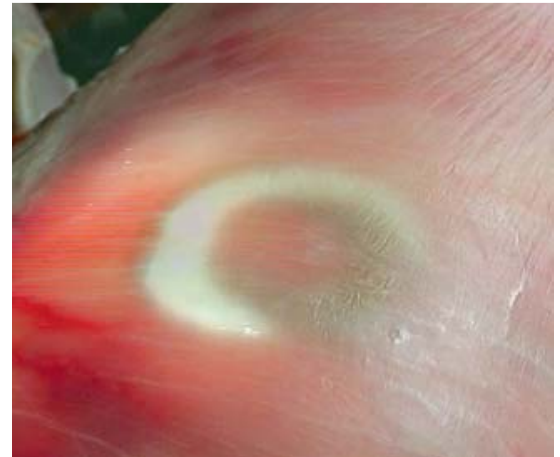

Fig. 2 The transmitted light from the endoscope (so-called light ring) allows the lesion to be detected through the thin muscular layer accurately.
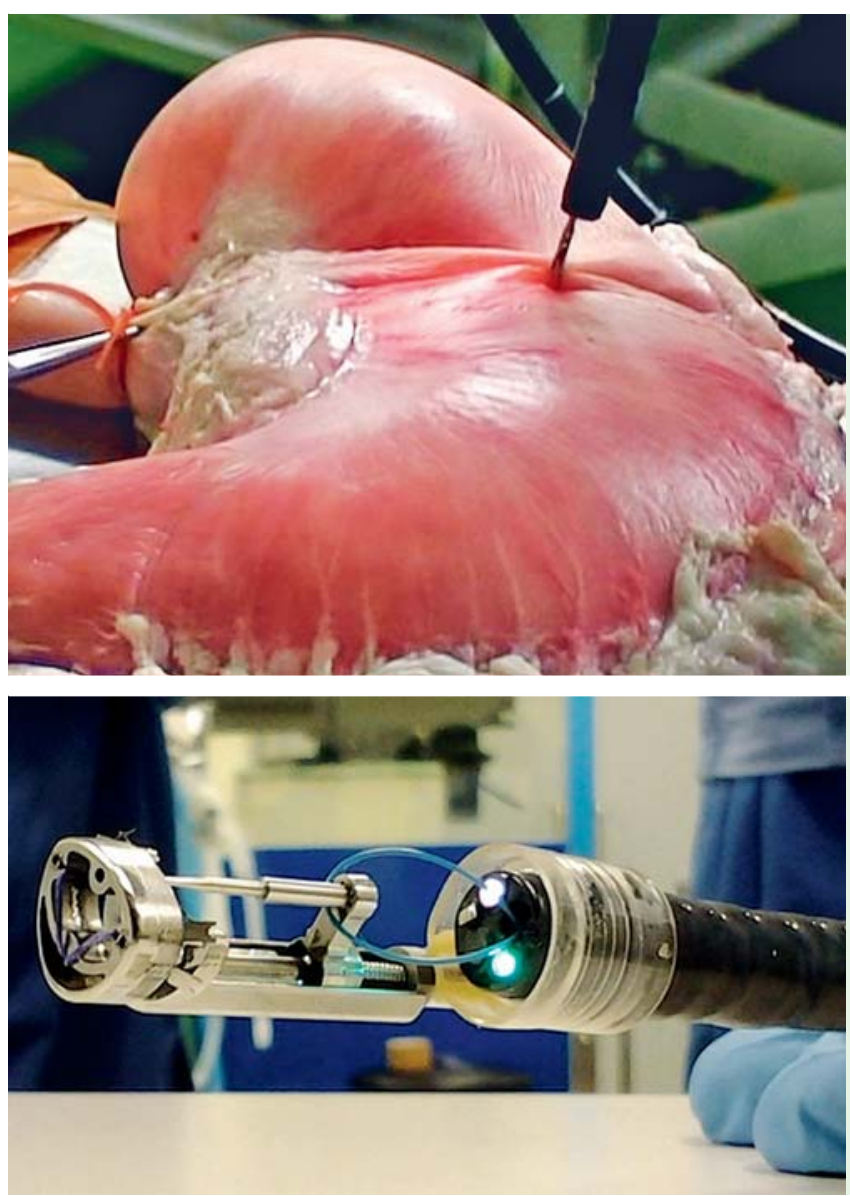

Fig. 3 The OTSC Twin Grasper is used to grasp and draw both sides of the light ring together, enabling us to invert the lesion into the stomach accurately.

Fig. 4 Full-thickness suturing is performed with another endoscope placed through the mouth and equipped with a suturing device (double-arm bar suturing system).
Although laparoscopic inverted endoscopic full-thickness resection (EFTR) has the advantage of nonexposure of the peritoneal cavity to gastric juice and tumor, like nonexposed wall inversion surgery (NEWS) [1] or the combination of laparoscopic and endoscopic approaches to neoplasia with a nonexposure technique (CLEAN-NET) [2], five ports are necessary to make hand-sewn sutures laparoscopically. We have developed a nonexposure EFTR technique that, with the use of a flexible endoscopic suturing device ( $\bullet$ Video 1 ), requires only one port and full-thickness suture.

After a pseudolesion with a 40-mm diameter was marked in an excised porcine stomach, a circular ring having a width of $8 \mathrm{~mm}$ that reached the muscularis propria was created by using the endoscopic submucosal dissection (ESD) technique around the pseudolesion ( $\bullet$ Fig. 1). A flexible endoscope was virtually inserted through the virtual $10-\mathrm{mm}$ umbilical port and advanced to the lesion from outside the stomach. The transmitted light, the so-called light ring ( $\bullet$ Fig. 2 ), was used to detect the lesion accurately. The use of a flexible endoscope through the virtual port to grasp and draw both sides of the light ring together with an OTSC Twin Grasper (Ovesco Endoscopy, Tübingen, Germany) enabled us to invert the lesion into the stomach accurately ( $\bullet$ Fig. 3 ).

Full-thickness suturing was performed by using another endoscope, equipped with a double-arm bar suturing system (DBSS), through the virtual mouth $[3,4]$ ( $\bullet$ Fig. 4). One end of the first arm of the DBSS was placed behind the inverted gastric wall. The second arm (the puncture needle) was moved forward and penetrated the full thickness of the inverted gastric wall. With this one step, the absorbable thread could be passed through the full thickness of the inverted gastric wall. The detainment snare was gradually contracted, and ligation was performed ( $\mathbf{F i g . 5}$ ). After suturing, full-thickness resection was performed from inside the stomach. An air leak test was conducted, showing $760-\mathrm{PaG}$ (Pascal gauge) pressure resistance ( $\bullet$ Fig. 6, $\bullet$ Video 2 ).

Non-exposed EFTR is a super-minimally invasive technique for partial gastrectomy that can be completed by using only flexible endoscopes.

Endoscopy_UCTN_Code_TTT_1AO_2AG 


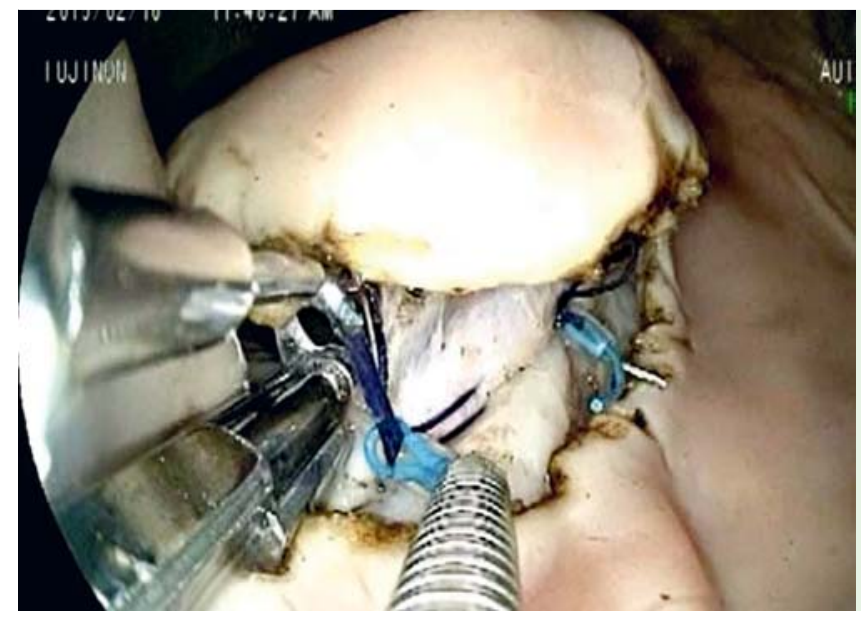

Fig. 5 The double-arm bar suturing system is used to create stitches with a 3-mm pitch.

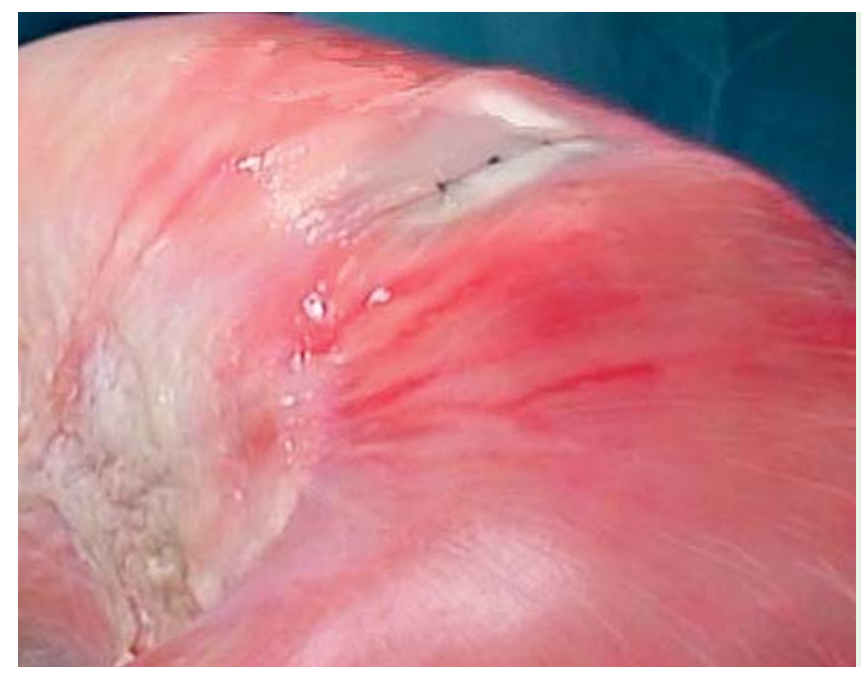

Fig. 6 An air leak test shows 760-PaG (Pascal gauge) pressure resistance.

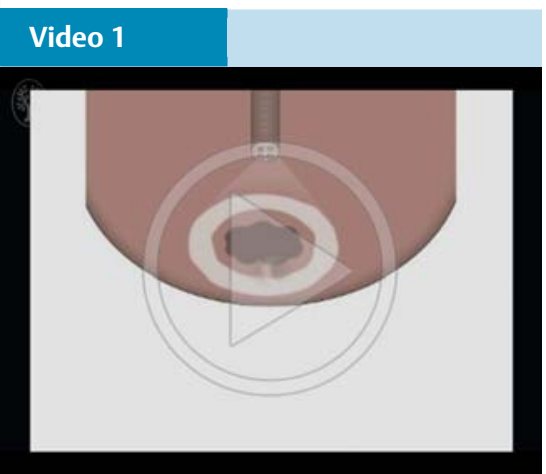

Conceptual images of nonexposure endoscopic full-thickness resection.

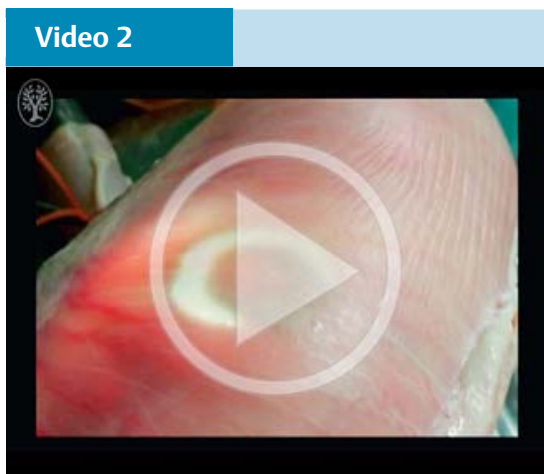

Nonexposure endoscopic full-thickness resection of a 40-mm-diameter pseudolesion in an excised porcine stomach.
Competing interests: The authors have declared no conflict of interest related to this article.

\section{Hirohito Mori, Hideki Kobara, Noriko Nishiyama, Shintaro Fujihara, Nobuya Kobayashi, Maki Ayaki, Tsutomu Masaki}

Department of Gastroenterology and Neurology, Kagawa University, Kagawa, Japan

\section{References}

1 Goto O, Mitsui T, Fujishiro M et al. New method of endoscopic full-thickness resection: a pilot study of non-exposed endoscopic wall-inversion surgery in an ex vivo porcine model. Gastric Cancer 2011; 14: 183-187

2 Inoue H, Ikeda H, Hosoya $T$ et al. Endoscopic mucosal resection, endoscopic submucosal dissection, and beyond: full-layer resection for gastric cancer with nonexposure technique (CLEAN-NET). Surg Oncol Clin N Am 2012; $21: 129-140$

3 Mori H, Kobara H, Kazi $R$ et al. Balloonarmed mechanical counter traction and double-armed bar suturing systems for pure endoscopic full-thickness resection. Gastroenterology 2014; 147: 278 - 280

4 Mori $H$, Kobara H, Rafiq K et al. New flexible endoscopic full-thickness suturing device: a triple-arm-bar suturing system. Endoscopy 2013; 45: 649-154

\section{Bibliography}

DOI http://dx.doi.org/

10.1055/s-0034-1393153

Endoscopy 2015; 47: E501-E502

(c) Georg Thieme Verlag KG

Stuttgart · New York

ISSN 0013-726X

\section{Corresponding author}

\section{Hirohito Mori, MD, PhD}

Department of Gastroenterology and Neurology Kagawa University

1750-1 Ikenobe

Miki, Kita

Kagawa 761-0793

Japan

Fax: +81-87-891-2158

hiro4884@med.kagawa-u.ac.jp 